\title{
Symmetry problem 1
}

\author{
Alexander G. Ramm \\ Mathematics Department, Kansas State University, \\ Manhattan, KS 66506-2602, USA \\ ramm@math.ksu.edu \\ http://www.math.ksu.edu/ ramm
}

\begin{abstract}
A symmetry problem is solved. A new method is used. The idea of this method is to reduce to a contradiction the PDE and the over-determined boundary data on the boundary.

The new method allows one to solve other symmetry problems.
\end{abstract}

\section{Introduction}

Symmetry problems for PDE were studied in many publications by many authors, see, for example, [1]. In this paper a new method is given for a study of symmetry problems for PDE. Throughout we assume that $D$ is a bounded connected $C^{2}$-smooth domain in $\mathbb{R}^{3}, S$ is the boundary of $D, N$ is the unit normal to $S$, pointing out of $D, u_{N}$ is the normal derivative of $u$ on $S, D^{\prime}=\mathbb{R}^{3} \backslash D, S^{2}$ is the unit sphere in $\mathbb{R}^{3}, J_{n}(r)$ is the Bessel function regular at $r=0, j_{\ell}(r)$ is the spherical Bessel function, $j_{\ell}^{\prime}(k r)=\frac{d j_{\ell}(k r)}{d r}, k>0$ is a constant, $\beta \cdot y=(\beta, y)$ is the dot product.

In $[2]-[10]$ the author studied various symmetry problems.

Let us formulate the symmetry problem studied in this paper. Our main result is formulated in Theorem 1.

Theorem 1. Assume that

$$
\Delta u+k^{2} u=0 \quad \text { in } \quad D,\left.\quad u\right|_{S}=1, \quad u_{N}=0 .
$$

Then $S$ is a sphere of radius a where a solves the equation $j_{0}^{\prime}(k a)=0$.

In [5], [7] it was shown that the Pompeiu problem is equivalent to the problem (1). In Section 2 proofs are given.

MSC: 35J05, 35R30

Key words: symmetry problems for PDE

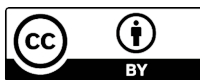




\section{Proofs}

Proof of Theorem 1. Let $g(x, y, k):=\frac{e^{i k|x-y|}}{4 \pi|x-y|}$. If problem (1) has a solution then this solution is unique by the uniqueness of the solution to the Cauchy problem for elliptic equation (1). The solution to equation (1) by Green's formula is:

$$
u(x)=-\int_{S} g_{N}(x, t) d t, \quad x \in D ; \quad u(x)=-\int_{S} g_{N}(x, t) d t=0, \quad x \in D^{\prime} .
$$

Let $B_{R}=\{x:|x| \leq R\}, D \subset B_{R}$. If $D$ is a ball $B_{a}$ of radius $a$ and $j_{0}^{\prime}(k a)=0$, then problem (1) in $B_{a}$ has a solution:

$$
u=\frac{j_{0}(k r)}{j_{0}(k a)}, \quad r=|x|
$$

In what follows we assume that $D \subset \mathbb{R}^{2}$ and $S$ is a closed smooth curve. Let $\mathbf{r}(\mathbf{s})=$ $x(s) e_{1}+y(s) e_{2}$ be a parametric representation of $S, s$ be the arc length along $S$ and also the corresponding to the arc length $s$ point on $S,\left\{e_{1}, e_{2}\right\}$ is a Cartesian basis in $\mathbb{R}^{2}$. The first boundary condition in $(1)$ is $u(x(s), y(s))=1$. Differentiating with respect to $s$ one gets $u_{x} \dot{x}+u_{y} \dot{y}=0$ and another differentiation yields

$$
u_{x x} \dot{x}^{2}+2 u_{x y} \dot{x} \dot{y}+u_{y y} \dot{y}^{2}=0, \quad \dot{x}=\frac{d x}{d s} .
$$

Here we used the formula $u_{x} \ddot{x}+u_{y} \ddot{y}=0$. This formula can be derived as follows: $\nabla u \cdot \ddot{\mathbf{r}}=\nabla u \cdot \kappa \nu$, where $\kappa=\kappa(s)>0$ is the curvature of $S, \nu=-N$ is the unit normal pointing into $D, \nabla u \cdot \nu=-u_{N}=0$ on $S$. From (1) it follows that

$$
u_{x x}+u_{y y}=-k^{2} \quad \text { on } \quad S .
$$

Let us prove that (4) and (5) are not compatible at some points, except when $S$ is a circle of radius $a$, where $a$ solves the equation $j_{0}^{\prime}(k a)=0$.

Denote $u_{x x}=p=p(s), u_{x y}=q=q(s)$. Then (5) implies $u_{y y}=-k^{2}-p$ on $S$. Let $A$ be a $2 \times 2$ matrix with elements $A_{11}=p, A_{12}=A_{21}=q, A_{22}=-k^{2}-p$. The equation for finding the eigenvalues $\lambda_{1,2}$ of $A$ is:

$$
\lambda^{2}+k^{2} \lambda-p^{2}-q^{2}-k^{2} p=0 .
$$

The eigenvalues $\lambda_{1}$ and $\lambda_{2}$ are:

$$
\lambda_{1,2}=-\frac{k^{2}}{2} \pm\left(k^{4} / 4+p^{2}+q^{2}+k^{2} p\right)^{1 / 2} .
$$

Clearly, $\lambda_{1}+\lambda_{2}=-k^{2}, \lambda_{1} \lambda_{2}=-p^{2}-q^{2}-k^{2} p, k^{4} / 4+p^{2}+q^{2}+k^{2} p=\left(\frac{k^{2}}{2}+p\right)^{2}+q^{2} \geq 0$. Thus, $\lambda_{2}<0$. 
The corresponding eigenvectors (non-normalized but orthogonal) can be calculated explicitly. One has

$$
e_{1}=\{1, \gamma\}, \quad \gamma:=\frac{q}{k^{2}+p+\lambda_{1}}=\frac{\lambda_{1}-p}{q}
$$

If $q \neq 0$, then

$$
e_{2}=\left\{\frac{k^{2}+p+\lambda_{2}}{q}, 1\right\}=\{-\gamma, 1\}
$$

If $q \neq 0$ then one checks that $\frac{k^{2}+p+\lambda_{2}}{q}=\frac{q}{\lambda_{2}-p}$ and $\frac{q}{\lambda_{2}-p}+\frac{q}{k^{2}+p+\lambda_{1}}=0$, so $\gamma=-\frac{k^{2}+p+\lambda_{2}}{q}$.

If $q=0$ then $\lambda_{1}=p, \lambda_{2}=-p-k^{2}, e_{1}=\{1,0\}, e_{2}=\{0,1\}$, and equation (13) (see below) leads also to a contradiction as in the case $q \neq 0$.

Clearly, $e_{1} \cdot e_{2}=0,\left\|e_{1}\right\|^{2}=\left\|e_{2}\right\|^{2}=1+\gamma^{2}$, so $\gamma^{2}$ is invariant under rotations of the Cartesian coordinate system.

Denote $\{\dot{x}, \dot{y}\}:=w$. Note that $\dot{x}^{2}+\dot{y}^{2}=1$. Let $c_{1}, c_{2}$ be scalar coefficients. Then

$$
c_{1} e_{1}+c_{2} e_{2}=w, \quad w:=\{\dot{x}, \dot{y}\}
$$

Solving explicitly this algebraic system for $c_{1}$ and $c_{2}$ one gets:

$$
c_{1}=(\dot{x}+\gamma \dot{y}) \Delta^{-1}, \quad \Delta=1+\gamma^{2},
$$

and

$$
c_{2}=(\dot{y}-\gamma \dot{x}) \Delta^{-1} .
$$

Equation (4) can be written as $(A w, w)=0$. Substitute $w$ from (10) into the equation $(A w, w)=0$ and use the orthogonality of $e_{1}$ and $e_{2}$ to get

$$
(\dot{y}-\gamma \dot{x})^{2} \lambda_{2}+(\dot{x}+\gamma \dot{y})^{2} \lambda_{1}=0 .
$$

We now prove that (13) leads to a contradiction unless $S$ is a circle of radius $a$ where $a$ solves the equation $J_{0}^{\prime}(k a)=0$ if $D \subset \mathbb{R}^{2}$ and $a$ solves the equation $j_{0}^{\prime}(k a)=0$ if $D \subset \mathbb{R}^{3}$.

Choose Cartesian coordinates in which $\dot{x}(s)=-\gamma \dot{y}$. Such coordinate system does exist because the only restriction on $\dot{x}$ and $\dot{y}$ is $\dot{x}^{2}+\dot{y}^{2}=1$ at all $s \in S$. Then, since $\lambda_{2}<0$ equation (13) with $\dot{x}(s)=-\gamma \dot{y}$ implies $\dot{y}\left(1+\gamma^{2}\right)=0$. Thus, $\dot{y}=0$. Therefore, $\dot{x}=\dot{y}=0$. This contradicts the relation $\dot{x}^{2}+\dot{y}^{2}=1$. This contradiction holds for any smooth $S$ except for a circle of a special radius, see (3).

Theorem 1 is proved. 


\section{References}

[1] T. Chatelain and A. Henrot, Some results about Schiffer's conjectures, Inverse Problems, 15, (1999), 647-658.

[2] A. G. Ramm, Inverse Problems, Springer, New York, 2005.

[3] A. G. Ramm, A symmetry problem, Ann. Polon. Math., 92, (2007), 49-54.

[4] A. G. Ramm, Symmetry problem, Proc. Amer. Math. Soc., 141, N2,(2013), 515-521.

[5] A. G. Ramm, The Pompeiu problem, Global Journ. of Math. Analysis (GJMA), 1, N1, (2013), 1-10. Open access Journal: http://www.sciencepubco.com/index.php/GJMA/issue/current

[6] A. G. Ramm, A symmetry result for strictly convex domains, Analysis, 35 (1), (2015), 29-32.

[7] A. G. Ramm, Solution to the Pompeiu problem and the related symmetry problem, Appl. Math. Lett., 63, (2017), 28-33.

[8] A. G. Ramm, Scattering by obstacles, D.Reidel, Dordrecht, 1986.

[9] A. G. Ramm, Scattering by obstacles and potentials, World Sci. Publ., Singapore, 2017.

[10] A. G. Ramm, Necessary and sufficient condition for a surface to be a sphere, Open J. Math. Anal., 2, (2018), issue $2,51-52$.

Open access: https://pisrt.org/psr-press/journals/oma/ 